\title{
AN ISOPERIMETRIC INEQUALITY FOR MOMENTS OF INERTIA OF PLANE CONVEX SETS
}

\author{
BY \\ TSUAN WU TING
}

1. Introduction. Let $\mathbf{K}$ be the class of all closed plane convex sets $K$ with given area $v$ and $C_{K}$ be the centroid of $K$. If the line $l(P, \theta)$ lies in the plane of $K$ through the point $P$ and with direction $\theta, 0 \leqq \theta \leqq 2 \pi$, we denote the moment of inertia of $K$ about the line $l(P, \theta)$ by $I(K, P, \theta)$. The maximum moment of inertia $I_{M}(K, P)$ and the minimum moment of inertia $I_{m}(K, P)$ about the point $P$ are defined by:

$$
\begin{aligned}
& I_{M}(K, P)=\max _{\theta} I(K, P, \theta)=I\left(K, P, \theta_{M}\right), \\
& I_{m}(K, P)=\min _{\theta} I(K, P, \theta)=I\left(K, P, \theta_{m}\right),
\end{aligned}
$$

where $\theta_{M}$ and $\theta_{m}$ are called the principal directions of the moments of inertia of $K$ with respect to the point $P$. It is known [1] that $I_{M}(K, P)$ and $I_{m}(K, P)$ exist for a given $K$ and a fixed point $P$, and $\left|\theta_{M}-\theta_{m}\right|=\pi / 2$.

The isoperimetric inequality to be established in this paper arises from the problem proposed by Truesdell and formulated by Keller, namely: find the shape of the strongest column with convex normal sections and with a given volume and height. To solve this extremal problem it is necessary to solve the following isoperimetric problem first: determine the shape of the convex set $K_{\Delta} \in \mathbf{K}$ such that

$$
I_{m}\left(K_{\triangle}, C_{K_{\Delta}}\right)=\max _{\mathbf{K}} I_{m}\left(K, C_{\mathbf{K}}\right)=\max _{\mathbf{K}} \min _{\theta} I\left(K, C_{K}, \theta\right) .
$$

This problem was investigated previously by Keller and others [2]. It was conjectured by Keller and Ungar that the solution figure is an equilateral triangle $K_{\Delta} \in \mathbf{K}$. Keller also pointed out that "there is little doubt" about the correctness of their conjecture and he used it to solve Truesdell's problem [2]. It is the purpose of this paper to give a proof of the conjecture. We now state it as

THEOREM. If $K \in \mathbf{K}$ then

$$
I_{m}\left(K, C_{K}\right)=\min _{\theta} I\left(K, C_{K}, \theta\right) \leqq \frac{v^{2}}{6 \sqrt{ } 3} .
$$

The equality sign holds, if, and only if, $K$ is an equilateral triangle $K_{\Delta} \in \mathbf{K}$.

Presented to the Society, April 12, 1962; received by the editors February 8, 1962 and, in revised form, June 14, 1962. 
As $I_{m}\left(K, C_{K}\right)$ is small if the diameter of $K$ is too large, we may consider only the sets of $\mathbf{K}$ with diameter less than a fixed number. Hence, the existence of solution follows from the Blaschke's compactness theorem [3]. The difficulty in proving our theorem is that convexity is a necessary condition for the existence of solution, but it is difficult to give it an analytic characterization so that Euler's variational technique would become applicable. Accordingly, the present proof is carried out by direct comparison of $I_{m}\left(K_{\Delta}, C_{K_{\Delta}}\right)$ with $I_{m}\left(K, C_{K}\right)$ for $K \in \mathbf{K}$. Therefore, this proof does not depend upon the existence proof of solution. It should be mentioned again that without loss of generality we shall restrict $\mathbf{K}$ to be the class of closed bounded plane convex sets with given area $v$.

2. Moment of inertia and area-preserving affine transformation. Since an affine transformation is linear, it preserves convexity. As we shall see, the area-preserving affine transformation will play an important role in this proof. First, we prove the following lemma which is simple and essential to further developments.

LEMMA 1. If $A$ is nonsingular affine transformation, then $A\left(C_{K}\right)=C_{A(K)}$. That is, the centroid is affinely invariant.

Proof. Let the matrix of $A$ relative to a fixed rectangular Cartesian coordinate system be

$$
\left(\begin{array}{ll}
a_{1} & b_{1} \\
a_{2} & b_{2}
\end{array}\right) .
$$

If $\left(x_{C_{K}}, y_{C_{K}}\right)$ are the coordinates of $C_{K}$, hen the coordinates of $A\left(C_{K}\right)$ are given by:

$$
\hat{x}_{c_{K}}=a_{1} x_{c_{K}}+b_{1} y_{c_{K}}, \hat{y}_{c_{K}}=a_{2} x_{c_{K}}+b_{2} y_{c_{K}} \text {. }
$$

On the other hand, the coordinates of $C_{A(K)}$ are defined by:

$$
\begin{aligned}
\hat{x}_{c_{A(K)}} & =\frac{1}{V(A(K))} \iint_{A(K)} \hat{x} d \hat{x} d \hat{y}=\frac{1}{V(K)} \iint_{K}\left(a_{1} x+b_{1} y\right) d x d y \\
& =a_{1} x_{C_{K}}+b_{1} y_{C_{K}}, \\
\hat{y}_{C_{A(K)}} & =\frac{1}{V(A(K))} \iint_{A(K)} \hat{y} d \hat{x} d \hat{y}=a_{2} x_{c_{K}}+b_{2} y_{c_{K}},
\end{aligned}
$$

where $V(A(K))$ and $V(K)$ denote the area of $A(K)$ and $K$ respectively. Now (1) and (2) indicate that $A\left(C_{K}\right)$ and $C_{A(K)}$ coincide and the lemma is proved.

To see the effect of an area-preserving affine transformation $A$ upon the moments of inertia $I\left(K, C_{K}, \theta\right)$ of a given set $K \in \mathbf{K}$, we prove the following two lemmas:

LEMMA 2. If $K \in \mathbf{K}$ and $I_{M}\left(K, C_{K}\right)>I_{m}\left(K, C_{K}\right)$, then there exists a set $A(K) \in \mathbf{K}$ such that 


$$
I_{m}\left(A(K), C_{A(K)}\right)>I_{m}\left(K, C_{K}\right) \text {. }
$$

Proof. Choose $C_{K}$ as the origin of a rectangular Cartesian coordinate system and choose the $x$ - and $y$-axes coincided with the principal directions of $I\left(K, C_{K}, \theta\right)$ so that

$$
I_{m}\left(K, C_{K}\right)=\int_{K} \int x^{2} d x d y, \quad I_{M}\left(K, C_{K}\right)=\int_{K} \int^{2} y^{2} d x d y, \quad \int_{K} \int x y d x d y=0 .
$$

Let

$$
\left(\begin{array}{ll}
a_{1} & b_{1} \\
a_{2} & b_{2}
\end{array}\right)
$$

be the matrix of an area-preserving affine transformation $A$ relative to the fixed coordinate system. It follows from Lemma 1 that

$$
\begin{aligned}
I\left(A(K), C_{A(K)}, 0\right) & =\int_{A(K)} \int_{y^{2}} d \hat{x} d \hat{y}=\iint_{K}\left(a_{2} x+b_{2} y\right)^{2} d x d y \\
& =a_{2}^{2} I_{m}\left(K, C_{K}\right)+b_{2}^{2} I_{M}\left(K, C_{K}\right), \\
I\left((A K), C_{A(K)}, \frac{\pi}{2}\right) & =\iint_{A(K)} \hat{x}^{2} d \hat{x} d \hat{y}=\iint_{K}\left(a_{1} x+b_{1} y\right)^{2} d x d y \\
& =a_{1}^{2} I_{m}\left(K, C_{K}\right)+b_{1}^{2} I_{M}\left(K, C_{K}\right),
\end{aligned}
$$

and

$$
\int_{A(K)} \int_{\hat{x})} \hat{y} d \hat{x} d \hat{y}=a_{1} a_{2} I_{m}\left(K, C_{K}\right)+b_{1} b_{2} I_{M}\left(K, C_{K}\right) .
$$

So far $A$ is only restricted to be area-preserving, i.e.,

$$
\operatorname{det}(A)=-b_{1} a_{2}+a_{1} b_{2}=1 .
$$

We may, therefore, require that

$$
a_{1} a_{2} I_{m}\left(K, C_{K}\right)+b_{1} b_{2} I_{M}\left(K, C_{K}\right)=0 .
$$

That is, the $x$ - and $y$-axes of the same coordinate system are also the principal directions of $I\left(A(K), C_{A(K)}, \theta\right)$. From (6) and (7), we have

$$
a_{2}=\frac{-b_{1} \beta}{a_{1}^{2}+b_{1}^{2} \beta}, \quad b_{2}=\frac{a_{1}}{a_{1}^{2}+b_{1}^{2} \beta},
$$

where

$$
\beta \equiv I_{M}\left(K, C_{K}\right) / I_{m}\left(K, C_{K}\right) \equiv 1+\varepsilon, \quad \varepsilon>0 .
$$

It may be noted that $\beta>1$ and the denominator in (8) does not vanish, if

$$
a_{1}^{2}+b_{1}^{2} \neq 0 \text {. }
$$


From (3), (4) and (8), we see that the affine image $A(K)$ of $K$ will be the set $A(K)$ to be exhibited if, and only if, the following two inequalities are satisfied:

$$
\begin{aligned}
a_{1}^{2}+b_{1}^{2} \beta & >1, \\
b_{1}^{2} \beta^{2}+a_{1}^{2} \beta & >\left(a_{1}^{2}+b_{1}^{2} \beta\right)^{2}, \quad \text { or } \beta>a_{1}^{2}+b_{1}^{2} \beta .
\end{aligned}
$$

That is, if

$$
1+\varepsilon>a_{1}^{2}+b_{1}^{2}+b_{1}^{2} \varepsilon>1,
$$

where we have made use of (9) and the fact $a_{1}^{2}+b_{1}^{2} \beta>0$. Now if we put

$$
a_{1}^{2}+b_{1}^{2}=1, \quad 0<b_{1}^{2}<1,
$$

then the inequalities in (11) are automatically satisfied and hence our lemma is proved.

From now on we denote by $J(K, P)$ the polar moment of inertia of $K$ about the line through the point $P$ and perpendicular to the plane of $K$. Also we note that

$$
J(K, P)=I(K, P, \theta)+I\left(K, P, \theta+\frac{\pi}{2}\right), \quad 0 \leqq \theta \leqq 2 \pi .
$$

Lemma 3. If $K \in \mathbf{K}, I_{m}\left(K, C_{K}\right)=I_{M}\left(K, C_{K}\right)=I$, then for every area-preserving affine transformation $A$ with $C_{K}$ as fixed point,

$$
J\left(A(K), C_{A(K)}\right) \geqq J\left(K, C_{K}\right) .
$$

Proof. Let

$$
\left(\begin{array}{ll}
a_{1} & b_{1} \\
a_{2} & b_{2}
\end{array}\right)
$$

be the matrix of $A$ relative to a fixed coordinate system with $C_{K}$ as origin. Proceeding precisely in the same way as what we have done in the previous proof, we obtain

$$
\begin{aligned}
-b_{1} a_{2}+a_{1} b_{2} & =1, \\
I\left(A(K), C_{A(K)}, 0\right) & =\left(a_{2}^{2}+b_{2}^{2}\right) I, \\
I\left(A(K), C_{A(K)}, \frac{\pi}{2}\right) & =\left(a_{1}^{2}+b_{1}^{2}\right) I .
\end{aligned}
$$

It is geometrically clear that $J(K, P)$ is invariant under any rigid rotation about the line through the point $P$ and perpendicular to the plane of $K$. Furthermore

$$
J(K, P)=I_{m}(K, P)+I_{M}(K, P) .
$$

Thus to evaluate $J\left(A(K), C_{A(K)}\right)$, we may assume that the $x$ - and $y$-axes are also the principal axes of $I\left(A(K), C_{A(K)}, \theta\right)$. Hence, by (5) 


$$
\begin{aligned}
a_{1} a_{2}+b_{1} b_{2} & =0 \\
I_{m}\left(A(K), C_{A(K)}\right) & =\left(a_{2}^{2}+b_{2}^{2}\right) I, \\
I_{M}\left(A(K), C_{A(K)}\right) & =\left(a_{1}^{2}+b_{1}^{2}\right) I .
\end{aligned}
$$

Solving the first equation in (13) and the equation (14) for $a_{2}$ and $b_{2}$, inserting the resulting expressions for $a_{2}$ and $b_{2}$ into the first equation in (15) and then adding the corresponding sides of the equations in (15), we obtain

$$
J\left(A(K), C_{A(K)}\right)=\left(\frac{1}{a_{1}^{2}+b_{1}^{2}}+a_{1}^{2}+b_{1}^{2}\right) J\left(K, C_{K}\right) / 2 .
$$

This shows that

$$
J\left(A(K), C_{A(K)}\right) \geqq J\left(K, C_{K}\right),
$$

and the lemma is proved. It may be noted that the equality sign holds, if, and only if, $A$ is a rigid rotation about $C_{K}$. We shall also use this fact in proving the theorem.

3. An extremal problem in polar moments of inertia. In proving the theorem we shall use Lemma 6 heavily. However, to prove Lemma 6 the following two lemmas are going to be used.

LEMMA 4. Let $(-s, 0),(b, h),(c, h),(s, 0)$ with $s>0, c>b, h>0$ be respectively the coordinates of the vertices $A, B, C, D$ of a quadrilateral. If the point $E$ with coordinates $(\xi, h)$ and the point $\hat{P}_{s}$ with coordinates $(\alpha, \beta)$ are given and if $c>\xi>b, \beta \leqq 0$, then either

$$
J\left(\triangle A B D, \hat{P}_{s}\right)>J\left(\triangle A E D, \hat{P}_{s}\right), \text { or }
$$

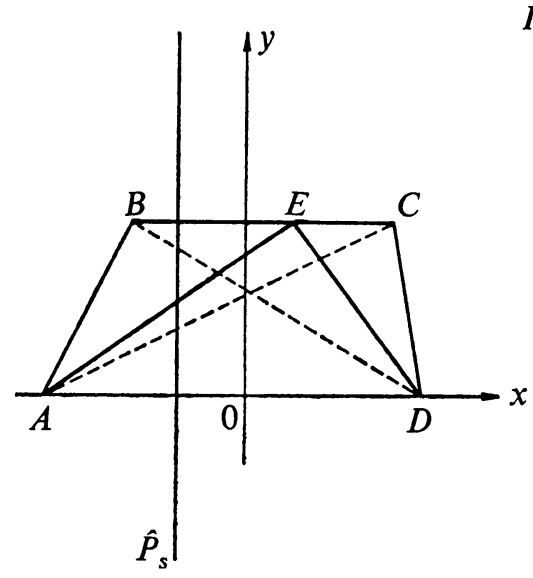

$$
J\left(\triangle A C D, \hat{P}_{s}\right)>J\left(\triangle A E D, \hat{P}_{s}\right)
$$

$$
I\left(\triangle A E D, \hat{P}_{s}, \frac{\pi}{2}\right)
$$

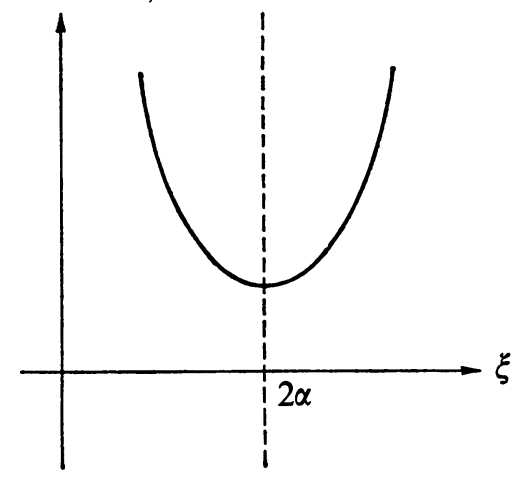

FIGURE 1 
Proof. First, we compare the moments of inertia of the three triangles about the line through the point $\hat{P}_{s}$, and perpendicular to $A D$. To this end we consider how $I\left(\triangle A E D, \hat{P}_{s}, \pi / 2\right)$ varies with the abscissa $\xi$ of the point $E$ when $E$ moves along the segment $B C$. To simplify computations we are going to use the following known results [1]: If a particle whose mass is one-third that of the triangle be placed at the middle of each side, the moment of inertia of the triangle about any line is the same as that of the system of the three particles. Clearly, the areas of the three triangles, $\triangle A E D, \triangle A B D$, and $\triangle A C D$, are all equal. Without loss of generality, we may assume the mass of each triangle to be 3 . Thus

$$
I\left(\triangle A E D, \hat{P}_{s}, \frac{\pi}{2}\right)=\alpha^{2}+\left(\alpha-\frac{\xi-s}{2}\right)^{2}+\left(\alpha-\frac{\xi+s}{2}\right)^{2} .
$$

That is, $I\left(\triangle A E D, \hat{P}_{s}, \pi / 2\right)$ as a function of $\xi$ for a fixed value of $\alpha$ is a concaveupward parabola. It achieves minimum at $\xi=2 \alpha$. Consequently, on any closed interval of $\xi$ its maximum is at the end point of the interval, so that we always have either

$$
\begin{aligned}
& I\left(\triangle A B D, \hat{P}_{s}, \frac{\pi}{2}\right)>I\left(\triangle A E D, \hat{P}_{s}, \frac{\pi}{2}\right), \text { or } \\
& I\left(\triangle A C D, \hat{P}_{s}, \frac{\pi}{2}\right)>I\left(\triangle A E D, \hat{P}_{s}, \frac{\pi}{2}\right) .
\end{aligned}
$$

Since the segments $A D$ and $B C$ are parallel,

$$
I(\triangle A E D, A, 0)=I(\triangle A B D, A, 0)=I(\triangle A C D, A, 0) .
$$

Moreover, the centroids of $\triangle A E D, \triangle A B D, \triangle A C D$ are collinear and they are on the line parallel to the segment $A D$. It follows from the "parallel-axis" theorem [1] that

$$
I\left(\triangle A B D, \hat{P}_{s}, 0\right)=I\left(\triangle A C D, \hat{P}_{s}, 0\right)=I\left(\triangle A E D, \hat{P}_{s}, 0\right) .
$$

Now (16) is a direct consequence of (17), (18) and (12). The lemma is now proved.

Lemma 5 is an approximation theorem of a special convex set by convex polygons, but the approximation polygons have to satisfy certain constraints. Since a theorem precisely of this form is not available, we state it as a lemma and omit the proof which is similar to those as given in [3]. In what follows, we use $\partial K$ to denote the frontier of $K$ and $V(K)$ the area of $K$. If $A, B$ are two points or one of them is a point, we use $\rho(A, B)$ to denote the usual distance between $A$ and $B$. If $K$ is a set, we use $U(K, \delta)$ to denote the set:

$$
U(K, \delta)=\{X \mid \rho(X, K)<\delta\} .
$$

Let $K_{1}, K_{2}$ be two convex sets. Let $\delta_{1}$ be the lower bound of the positive numbers $\delta$ such that $U\left(K_{1}, \delta\right) \supset K_{2}$ and $\delta_{2}$ be the lower bound of the positive numbers $\delta$ 
such that $U\left(K_{2}, \delta\right) \supset K_{1}$. The distance $\rho^{*}$ between the two convex sets $K_{1}, K_{2}$ is defined by the formula:

$$
\rho^{*}\left(K_{1}, K_{2}\right)=\delta_{1}+\delta_{2} .
$$

LEMma 5. If $\hat{K} \in \mathbf{K}, \partial \hat{R}$ contains the segments $\hat{P}_{s} P_{1}$ and $\hat{P}_{s} P_{2}$ and no larger segments $\hat{P}_{s} P_{1}^{*}, \hat{P}_{s} P_{2}^{*}$ and if $S_{1}, S_{2}$ are support lines of $\hat{K}$ at $P_{1}, P_{2}$ respectively, then for every $\varepsilon>0$, there is a polygon $H$ with the properties: (i) $\rho^{*}(\hat{K}, H)<\varepsilon$, (ii) $\partial H$ contains $\hat{P}_{s} P_{1}, \hat{P}_{s} P_{2}$, (iii) $S_{1}, S_{2}$ are lines of support of $H$ at $P_{1}, P_{2}$ respectively.

Lemma 6. Let $\triangle \hat{P}_{s} A_{1} A_{2}$ be a triangle with $\rho\left(\hat{P}_{s}, A_{1}\right)>\rho\left(\hat{P}_{s}, A_{2}\right)$ and let $A_{1}^{*}$ be the point on $\hat{P}_{s} A_{1}$ and $\rho\left(\hat{P}_{s} A_{1}^{*}\right)=\rho\left(\hat{P}_{s}, A_{2}\right)$. If $\hat{R}$ is a convex set with area equal to that of $\triangle \hat{P}_{s} A_{1} A_{2}$ and $\partial \hat{R} \supset \hat{P}_{s} A_{2}, \partial R \supset \hat{P}_{s} A_{1}^{*}$, then

$$
J\left(\hat{K}, \hat{P}_{s}\right)<J\left(\Delta \hat{P}_{s} A_{1} A_{2}, \hat{P}_{s}\right)
$$

unless $\hat{R}$ is the triangle $\hat{P}_{s} A_{1} A_{2}$ or its mirror image.

Proof. First we show that the triangle $\hat{P}_{s} A_{1} A_{2}$ has greater polar moment of inertia than that of any other triangles which have the same area and which contain $\hat{P}_{s} A_{2}$ and $\hat{P}_{s} A_{1}^{*}$ in their sides. Consider any such triangle $\hat{P}_{s} A_{1}^{\prime \prime} A_{2}^{\prime \prime}$. Put

$$
\rho\left(\hat{P}_{s}, A_{2}\right)=b, \quad \rho\left(\hat{P}_{s}, A_{1}\right)=c, \quad \rho\left(\hat{P}_{s}, A_{2}^{\prime \prime}\right)=b^{\prime \prime}, \quad \rho\left(\hat{P}_{s}, A_{1}^{\prime \prime}\right)=c^{\prime \prime},
$$

and $\angle A_{1} \hat{P}_{s} A_{2}=\theta$. By hypothesis

$$
b^{\prime \prime} \geqq b, \quad c^{\prime \prime} \geqq b,
$$

and

$$
b c \sin \theta=b^{\prime \prime} c^{\prime \prime} \sin \theta
$$

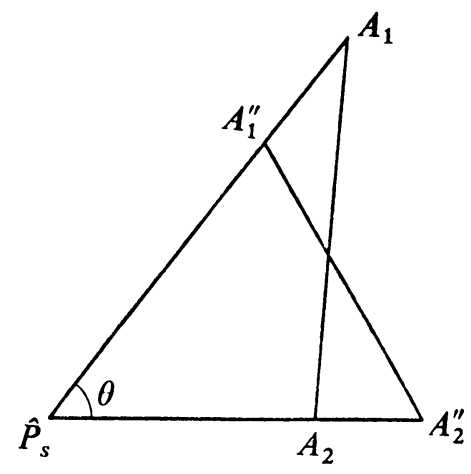

FIGURE 2

Straight-forward computations analogous to what has been done in Lemma 4 give 


$$
\begin{aligned}
& J\left(\triangle \hat{P}_{s} A_{1} A_{2}, \hat{P}_{s}\right)=\frac{1}{2}\left(b^{2}+c^{2}-2 b c \cos \theta\right), \\
& J\left(\triangle \hat{P}_{s} A_{1}^{\prime \prime} A_{2}^{\prime \prime}, \hat{P}_{s}\right)=\frac{1}{2}\left(b^{\prime \prime 2}+c^{\prime \prime 2}-2 b^{\prime \prime} c^{\prime \prime} \cos \theta\right) .
\end{aligned}
$$

It follows from (23) and (24) that

if, and only if,

$$
J\left(\triangle \hat{P}_{s} A_{1} A_{2}, \hat{P}_{s}\right) \geqq J\left(\Delta \hat{P}_{s} A_{1}^{\prime \prime} A_{2}^{\prime \prime}, \hat{P}_{s}\right),
$$

$$
b^{2}+c^{2} \geqq b^{\prime \prime 2}+c^{\prime \prime 2} .
$$

In view of (23), the inequality in (25) holds, if and only if,

$$
b+c \geqq b^{\prime \prime}+c^{\prime \prime}
$$

That is,

$$
b^{\prime \prime} \leqq c .
$$

But this inequality is a direct consequence of (23) and the second inequality in (22). Thus our lemma is proved for the case of triangles.

If $\hat{K}$ is not a triangle, let $\hat{P}_{s} A_{1}^{\prime}, \hat{P}_{s} A_{2}^{\prime}$ be the maximum linear segments of $\partial \hat{K}$ through the point $\hat{P}_{s}$. By hypothesis, we have

$$
\rho\left(\hat{P}_{s}, A_{1}^{\prime}\right) \geqq \rho\left(\hat{P}_{s}, A_{2}^{*}\right), \quad \rho\left(\hat{P}_{s}, A_{2}^{\prime}\right) \geqq \rho\left(\hat{P}_{s}, A_{2}\right) .
$$

Let the point $A \in \partial \hat{K}$ be such that a line of support of $\hat{K}$ at $A$ does not meet $\hat{P}_{s} A_{1}^{\prime}$ and $\hat{P}_{s} A_{2}^{\prime}$. If both the arcs $A A_{1}^{\prime}, A A_{2}^{\prime}$ of $\partial \hat{K}$ are linear segments, then the lemma follows by a single application of Lemma 4 . For if we move the point $A$ along the line parallel to the segment $A_{1}^{\prime} A_{2}^{\prime}$ until it just meets the extension of $\hat{P}_{s} A_{1}^{\prime}$ or $\hat{P}_{s} A_{2}^{\prime}$, then $\hat{K}$ is deformed into a triangle with the same area and the polar moment of inertia of this triangle about $\hat{P}_{s}$ is greater than $J\left(\hat{K}, \hat{P}_{s}\right)$ but less than $J\left(\triangle \hat{P}_{s} A_{1} A_{2}, \hat{P}_{s}\right)$. Otherwise, we assume first $\hat{K}$ to be a polygon. Now $\hat{P}_{s} A$ separates $\hat{K}$ into two convex polygons $\hat{K}_{1}, \hat{K}_{2}$ which contain the points $A_{1}^{\prime}, A_{2}^{\prime}$ respectively. If $\hat{K}_{1}$ has more than 4 vertices, we repeatedly apply Lemma 4 to reduce $\hat{K}_{1}$ to new polygons in such a way that (i) each of the new convex polygons with $\hat{P}_{s}, A_{1}^{\prime}$, $A$ as vertices and with area equal to $V\left(\hat{K}_{1}\right)$, (ii) each of the new polygons has greater polar moment of inertia about $\hat{P}_{s}$ and has one vertex less than the previous one. Similarly, if $\hat{K}_{2}$ has more than 4 vertices, we apply Lemma 4 to make the same reduction. In this way, we shall arrive at the single convex polygon $\hat{K}^{\prime}, \hat{P}_{s} A_{1}^{\prime} A_{1}^{\prime \prime} A A_{2}^{\prime \prime} A_{2}^{\prime} \hat{P}_{s}$, such that

$$
V\left(\triangle A_{1}^{\prime} A_{1}^{\prime \prime} A\right)=V\left(\hat{K}_{1}-\triangle \hat{P}_{s} A_{1}^{\prime} A\right), V\left(\triangle A_{2}^{\prime} A_{2}^{\prime \prime} A\right)=V\left(\hat{K}_{2}-\triangle \hat{P}_{s} A_{2}^{\prime} A\right),
$$

and the points $A_{1}^{\prime \prime}, A_{2}^{\prime \prime}$ lie somewhere on the segments $B_{1}^{\prime} B_{1}^{\prime \prime} / / A_{1}^{\prime} A, B_{2}^{\prime} B_{2 /}^{\prime \prime} / A_{2}^{\prime} A$ respectively. From the very method of reduction, we have

$$
J\left(\hat{R}, \perp_{e}\right)<J\left(\hat{R}^{\prime}, \hat{P}_{s}\right) .
$$




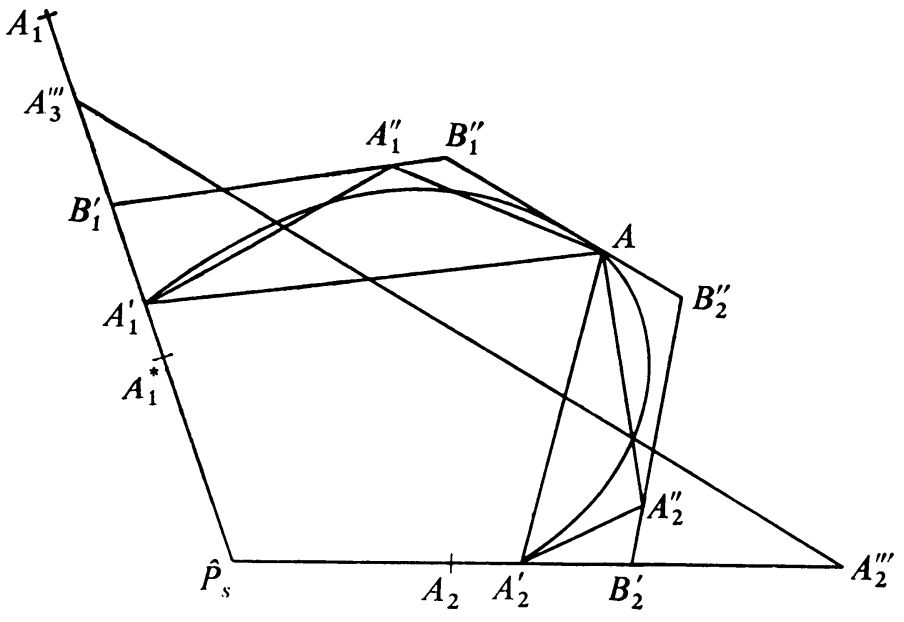

FIGURE 3

In the general case, we apply Lemma 5 to approximate $\hat{K}_{1}, \hat{K}_{2}$ by convex polygons with sufficient accuracy and then repeatedly apply Lemma 4 to these polygons in the way as just described. This will lead to a similar polygon $\hat{K}^{\prime}$. Furthermore, a limiting process based on Lemma 5 ensures that

$$
J\left(\hat{K}, \hat{P}_{s}\right) \leqq J\left(\hat{K}^{\prime}, \hat{P}_{s}\right),
$$

provided the approximations of $\hat{K}_{1}, \hat{K}_{2}$ by polygons are sufficiently close. Since $\hat{K}^{\prime}$ has at least 4 vertices and at most 6 vertices, we apply now Lemma 4 to $\hat{K}^{\prime}$ once, twice or three times and we come to a triangle $\hat{P}_{s} A_{1}^{\prime \prime \prime} A_{2}^{\prime \prime \prime}$ such that

$$
\rho\left(\hat{P}_{s}, A_{1}^{\prime \prime \prime}\right) \geqq \rho\left(\hat{P}_{s}, A_{1}^{\prime}\right), \quad \rho\left(\hat{P}_{s}, A_{2}^{\prime \prime \prime}\right) \geqq \rho\left(\hat{P}_{s}, A_{2}^{\prime}\right), \quad V\left(\Delta \hat{P}_{s} A_{1}^{\prime \prime \prime} A_{2}^{\prime \prime \prime}\right)=V(\hat{K}),
$$

and

$$
J\left(\hat{K}, \hat{P}_{s}\right)<J\left(\Delta \hat{P}_{s} A_{1}^{\prime \prime \prime} A_{2}^{\prime \prime \prime}, \hat{P}_{s}\right) .
$$

The lemma now follows from the proof for the case of triangles.

4. Proof of theorem. By establishing the previous lemmas we are now ready to prove the theorem. By Lemma 2 the solution figure to the isoperimetric problem must have the property that its moment of inertia about all lines in its plane and passing through its centroid are all equal. Accordingly, we may restrict $\mathbf{K}$ to be the class of convex sets with this additional property.

Let $K \in \mathbf{K}$ with $I_{m}\left(K, C_{K}\right)=I_{M}\left(K, C_{K}\right)$. The convexity of $K$ implies the existence of a six-partite point $[3 ; 4 ; 5]$. We denote this point by $P_{s}$. If $C_{K}$ is a sixpartite point, we always choose $C_{K}$ as the point $P_{s}$ under consideration. Also we choose $C_{K}$ as the fixed point of an affine transformation. Under an area-preserving affine transformation $A$, the image $\hat{P}_{s}=A\left(P_{s}\right)$ will be the six-partite point of $\hat{K}=A(K)$. Furthermore, we adjust the transformation $A$ so that the three division lines, which pass through $\hat{P}_{s}$ and divide the area of $\hat{K}$ into six equal parts, will make an angle $\pi / 3$ with one another. 
We are going to compare $J\left(\hat{K}, \hat{P}_{s}\right)$ with $J\left(K_{\Delta}, C_{K_{\Delta}}\right)$ where $K_{\Delta}$ is an equilateral triangle in $\mathbf{K}$. To this end we denote in the clock-wise order the vertices of $K_{\triangle}$ by $A_{1}, A_{3}$ and $A_{5}$, the intersection points of $\partial \hat{K}$ and its division lines by $P_{1}, P_{2}, P_{3}, P_{4}, P_{5}$ and $P_{6}$, and the three medians of $K_{\triangle}$ by $A_{1} A_{4}, A_{2} A_{5}, A_{3} A_{6}$.

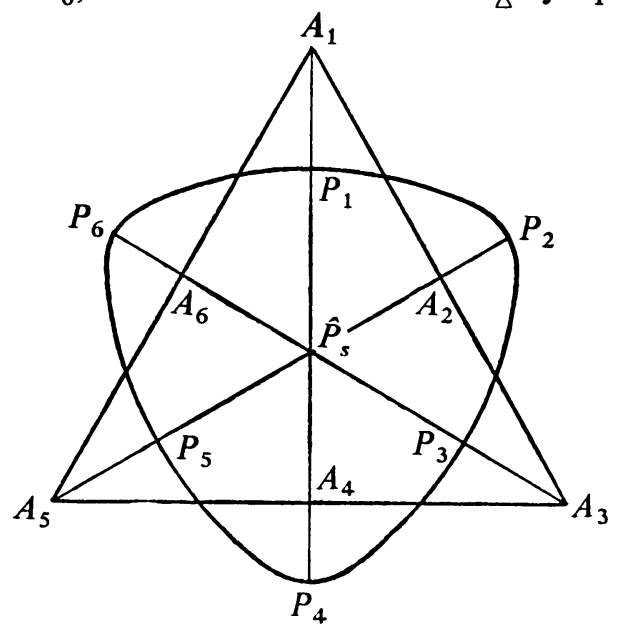

FIGURE 4

It may be noted that $C_{K_{\Delta}}$ is a six-partite point of $K_{\Delta}$ and the three medians divide the area of $K_{\Delta}$ into six equal parts. Place $K_{\Delta}$ upon $\hat{K}$ so that $C_{K_{\Delta}}$ coincides with $\hat{P}_{s}$ and the three medians $A_{1} A_{4}, A_{2} A_{5}, A_{3} A_{6}$ coincides with the division lines $P_{1} P_{4}, P_{2} P_{5}, P_{3} P_{6}$ respectively.

Now we assert that

$$
\rho\left(\hat{P}_{s}, P_{i}\right) \geqq h / 3, \quad i=1,2, \cdots, 6,
$$

where $h$ denotes the height of $K_{\Delta}$. To prove this assertion we denote by $R_{i, i+1}$ the the sector of $\hat{K}$ bounded by segments $\hat{P}_{s} P_{i}, \hat{P}_{s} P_{i+1}$ and $\partial \hat{K}$ for $i=1,2, \cdots, 5$, and denote by $\hat{K}_{1.6}$ the sector of $\hat{K}$ bounded by segments $\hat{P}_{s} P_{1}$ and $\hat{P}_{s} P_{6}$ and $\partial \hat{K}$. Suppose now $\rho\left(\hat{P}_{s}, P_{2}\right)<h / 3$. Since $V\left(\hat{K}_{1,2}\right)=V\left(\Delta \hat{P}_{s} A_{1} A_{2}\right)$, $V\left(\hat{K}_{2,3}\right)=V\left(\triangle \hat{P}_{s} A_{2} A_{3}\right)$, there are a point $P_{1.2} \in \hat{K}_{1.2}$ but not in $\triangle \hat{P}_{s} A_{1} A_{2}$ and a point $P_{2,3} \in R_{2,3}$ but not in $\triangle \hat{P}_{s} A_{2} A_{3}$. Now the convexity of $R$ implies that the triangle $\hat{P}_{s} P_{1,2} P_{2,3}$ is contained in $\hat{K}$. But $\triangle \hat{P}_{s} P_{1,2} P_{2,3}$ contains $P_{2}$ in its in terior. This contradicts that $P_{2} \in \partial R$. Hence $\rho\left(\hat{P}_{s}, P_{2}\right) \geqq h / 3$. Similar arguments prove that (26) holds for $i=4,6$. To prove (26) for $i=1,3,5$, we rotate $K_{\Delta}$ about the point $\hat{P}_{s}$ through an angle $\pi / 3$ and then apply the same arguments.

Consider now the triangle $\hat{P}_{s} A_{1} A_{2}$ and the convex set $\hat{K}_{1,2}$. The inequalities in (26) and the relation $V\left(\triangle \hat{P}_{s} A_{1} A_{2}\right)=V\left(\hat{K}_{1,2}\right)$ imply that $\hat{K}_{1,2}$ satisfies all the conditions in Lemma 6. Hence

$$
J\left(\hat{K}_{1,2}, \hat{P}_{s}\right) \leqq J\left(\triangle \hat{P}_{s} A_{1} A_{2}, \hat{P}_{s}\right),
$$

where the equality sign can hold only when $\hat{K}_{1.2}$ is $\Delta \hat{P}_{s} A_{1} A_{2}$ or its mirror image. 
Since Lemma 6 can be applied to any one of the six equal areas of $\mathbb{R}$, it follows that

$$
J\left(\hat{K}, \hat{P}_{s}\right) \leqq J\left(\triangle A_{1} A_{2} A_{3}, \hat{P}_{s}\right)=J\left(K_{\Delta}, C_{K_{\Delta}}\right),
$$

where the equality sign can hold only when $\hat{K}$ is $K_{\Delta}$. If $\hat{K}$ is $K_{\Delta}$, then $K=A^{-1}(\hat{K})$ is a triangle, $C_{K}, P_{s}, \hat{P}_{s}$ coincide by our choice of the transformation $A$. Hence, by Lemma 3 ,

$$
J\left(K, C_{K}\right) \leqq J\left(\hat{K}, \hat{P}_{s}\right)=J\left(K_{\Delta}, C_{K_{\Delta}}\right),
$$

where the equality sign can hold only when $K$ is $K_{\Delta}$. We need to consider the only case:

$$
J\left(\hat{R}, \hat{P}_{s}\right)<J\left(K_{\Delta}, C_{K_{\Delta}}\right) .
$$

By "Parallel-axis" theorem for polar moment of inertia,

$$
J\left(\hat{K}, C_{\hat{K}}\right) \leqq J\left(\hat{K}, \hat{P}_{s}\right),
$$

and the equality sign holds only if $\hat{P}_{s}$ is $C_{\hat{K}}$. Hence

$$
J\left(\hat{K}, C_{\hat{K}}\right)<J\left(K_{\Delta}, C_{K_{\Delta}}\right) .
$$

Since $\hat{K}$ is such that $I_{m}\left(K, C_{K}\right)=I_{M}\left(K, C_{K}\right)$, we have, by Lemma 3,

$$
J\left(K, C_{K}\right) \leqq J\left(\hat{K}, C_{\hat{K}}\right)<J\left(K_{\triangle}, C_{K_{\Delta}}\right)
$$

Thus,

$$
I_{m}\left(K, C_{K}\right)=\frac{1}{2} J\left(K, C_{K}\right)<\frac{1}{2} J\left(K_{\Delta}, C_{K_{\Delta}}\right)=I_{m}\left(K_{\Delta}, C_{K_{\Delta}}\right) .
$$

Clearly, $K$ is not $K_{\Delta}$ in this case. For if $K$ were $K_{\Delta}$, then the transformation $A$ becomes a rigid rotation and we must have $J\left(\hat{K}, \hat{P}_{s}\right)=J\left(K_{\Delta}, C_{K_{\Delta}}\right)$. The proof to our theorem is now completed.

ACKNOWLEDGEMENTs: The author wishes to express his thanks to the referee for his critical examinations and informative comments.

\section{REFERENCES}

1. E. J. Routh, Dynamics of a system of rigid bodies. I, Dover, New York, 1960; Chapter 1.

2. J. B. Keller, The shape of the strongest column, Arch. Rational Mech. Anal. 5 (1960), 275-285.

3. H. G. Eggleston, Convexity, Cambridge Univ. Press, Cambridge, 1958; Chapters 1, 4, 6.

4. R. C. Buck and E. F. Buck, Equipartition of convex sets, Math. Mag. 22 (1949), 195-198.

5. A. S. Besicovitch, Measure of asymmetry of convex curves, J. London Math. Soc. 23 (1948), 237-240.

The UNIVERSITY OF TEXAS, Austin, Texas 